TRANSACTIONS OF THE

AMERICAN MATHEMATICAL SOCIETY

Volume 353, Number 10, Pages 4083-4093

S 0002-9947(01)02843-4

Article electronically published on May 22, 2001

\title{
LIMITS IN THE UNIFORM ULTRAFILTERS
}

\author{
JONI BAKER AND KENNETH KUNEN
}

\begin{abstract}
Let $u(\kappa)$ be the space of uniform ultrafilters on $\kappa$. If $\kappa$ is regular, then there is an $\mathbf{x} \in u(\kappa)$ which is not an accumulation point of any subset of $u(\kappa)$ of size $\kappa$ or less. $\mathbf{x}$ is also good, in the sense of Keisler.
\end{abstract}

\section{INTRODUCTION}

If $\kappa$ is any infinite cardinal, we have $u(\kappa) \subseteq \kappa^{*} \subseteq \beta \kappa$. Here, $\kappa$ has the discrete topology, so its Cech compactification, $\beta \kappa$, is the space of ultrafilters on $\kappa$, and $\kappa^{*}=\beta \kappa \backslash \kappa$ is the the space of nonprincipal ultrafilters on $\kappa$. Then, we use $u(\kappa)$ to denote the space of uniform ultrafilters; that is, $\mathbf{x} \in u(\kappa)$ iff every set in $\mathbf{x}$ has size $\kappa$.

In studying limits, one is naturally led to $P$-points and weak $P$-points:

Definition 1.1. If $\theta$ is an infinite cardinal and $\mathbf{x}$ is a point in a topological space $X$, then:

- $\mathbf{x}$ is a $P_{\theta}$-point in $X$ iff the intersection of any family of fewer than $\theta$ neighborhoods of $\mathbf{x}$ is also a neighborhood of $\mathbf{x}$.

- $\mathbf{x}$ is a weak $P_{\theta}$-point in $X$ iff $\mathbf{x}$ is not a limit point of any subset of $X \backslash\{\mathbf{x}\}$ of size less than $\theta$.

So, a $P$-point is a $P_{\omega_{1}}$-point, and a weak $P$-point is a weak $P_{\omega_{1}}$-point. In any $T_{1}$ space, every $P_{\theta}$-point is a weak $P_{\theta}$-point. The main theorem of this paper is:

Theorem 1.2. If $\kappa$ is regular, then there is an $\mathbf{x} \in u(\kappa)$ which is a weak $P_{\kappa^{+}}$-point in $u(\kappa)$, and hence a weak $P_{\kappa}$-point in $\beta \kappa$.

This is best possible as a $Z F C$ result. Note that for $\mathbf{x} \in X \subseteq Y$, if $\mathbf{x}$ is a weak $P_{\theta}$-point in $Y$, then $\mathbf{x}$ is a weak $P_{\theta}$-point in $X$; but not conversely, in general. If

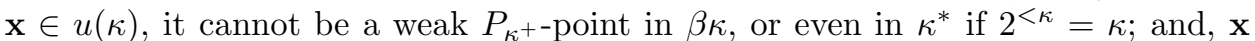
cannot be a weak $P_{\kappa^{++}}$-point in $u(\kappa)$ if $2^{\kappa}=\kappa^{+}$. The "hence" in the theorem is correct because by regularity of $\kappa$, no $\mathbf{x} \in u(\kappa)$ can be a limit of a subset of $\beta \kappa \backslash u(\kappa)$ of size less than $\kappa$.

For $\kappa=\omega$ (where $u(\omega)=\omega^{*}$ ), the theorem is already known (Kunen [6]). By a still earlier result of W. Rudin [7, it is consistent with $Z F C$ that there is even a $P$-point in $\omega^{*}$, although the existence of $P$-points is also independent (Shelah). The theorem for $\kappa=\omega$ can be improved in various models of $\neg C H$. For example, if

Received by the editors September 18, 2000 and, in revised form, March 21, 2001.

2000 Mathematics Subject Classification. Primary 54D80, 54D40.

Key words and phrases. Weak $P$-point, good ultrafilter, mediocre point.

Both authors' work was partly supported by NSF Grant DMS-9704520. They wish to thank the referee for a number of useful comments. 
$M A$ holds, then Rudin's proof yields a $P_{\mathfrak{c}}$-point (and hence a weak $P_{\mathfrak{c}}$-point) in $\omega^{*}$. However, it is consistent with $\mathfrak{c}$ arbitrarily large that there are no weak $P_{\omega_{2}}$-points in $\omega^{*}$ (add random reals or Cohen reals to a model of $\mathrm{CH}$ ).

For $\kappa>\omega$, we do not know about the consistency of possible strengthenings of this theorem (along the $\kappa=\omega$ lines), except for the following two elementary remarks: First, if $\kappa$ is regular and larger than $\omega$ but less than the first (2-valued) measurable cardinal, then there are no $P$-points in $u(\kappa)$. Second, it is consistent with $2^{\kappa} \gg \kappa^{+}$that there are no weak $P_{\kappa^{++}}$-points in $u(\kappa)$; just use Cohen forcing (with conditions of size less than $\kappa$ ) over a model of $G C H$.

We do not know if the theorem holds for singular $\kappa$. However, the existence of a weak $P_{\kappa}$-point in $\beta \kappa$ is already known, and this holds for singular $\kappa$ as well. In fact, it is well-known (see Section 2) that if $\mathbf{x} \in u(\kappa)$ is $\kappa^{+}$-good in the sense of Keisler, then $\mathbf{x}$ is a weak $P_{\kappa}$-point in $\beta \kappa$, and hence also in $u(\kappa)$. Such good ultrafilters exist (Keisler [4] under $G C H$ and Kunen [5] in $Z F C$ ). The $\mathbf{x}$ in Theorem 1.2] will be $\kappa^{+}$-good, and we shall add another ingredient to the inductive construction of [5]

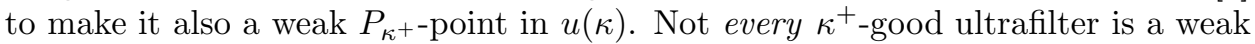
$P_{\kappa^{+}}$-point in $u(\kappa)$; for a counterexample, use the product of two good ultrafilters.

In Section 2, we first observe that the notion "good" is really a topological notion which makes sense for a point $\mathbf{x}$ in an arbitrary space $X$. Every $\kappa^{++}$-good point is a weak $P_{\kappa^{+}}$-point. However, one cannot prove in $Z F C$ that there is an $\mathbf{x} \in u(\kappa)$ which is $\kappa^{++}$-good in $u(\kappa)$ (Theorem 2.8). So, we shall weaken " $\kappa^{++}$-good" to " $\kappa^{+}$-mediocre". Every point which is both $\kappa^{+}$-mediocre and $\kappa^{+}$-good is a weak $P_{\kappa^{+}}$-point. The rest of the paper is then devoted to showing that such points exist in $u(\kappa)$.

The proof follows the standard pattern from [5] 6]; one constructs an ultrafilter in $2^{\kappa}$ steps with the aid of a matrix of sets. However, the matrix used here is a little more complicated than the standard ones, and it requires some argument to prove that it really exists. Section 3 proves some general results on constructing matrices, and Section 4 constructs the actual matrix we need. Then, Section 6 uses

this matrix to prove Theorem 1.2 Regularity of $\kappa$ is used only to prove that the matrix exists; the construction of the ultrafilter given the matrix works for any $\kappa$.

Notation: When discussing subsets of $\kappa, A \subseteq \subseteq^{*} B$ means that $|A \backslash B|<\kappa$, $A={ }^{*} B$ means that $A \subseteq{ }^{*} B \subseteq^{*} A$, and $A \perp B$ means that $|A \cap B|<\kappa . \mathcal{P}(\kappa) /<\kappa$ denotes the quotient algebra of $\mathcal{P}(\kappa)$ modulo the ideal $\{A \in \mathcal{P}(\kappa):|A|<\kappa\}$. Then $[A] \in \mathcal{P}(\kappa) /<\kappa$ is the equivalence class of $A \in \mathcal{P}(\kappa)$. For $\kappa=\omega$, we use $\mathcal{P}(\omega) /$ fin for $\mathcal{P}(\omega) /<\omega$.

\section{Good And Mediocre Points}

The following notation for intersections will be used throughout this paper:

Definition 2.1. Given sets $X_{\alpha}$ for $\alpha<\theta$ and $p \in[\theta]^{<\omega}: X_{[p}=\bigcap_{\alpha \in p} X_{\alpha}$.

Definition 2.2. A point $\mathbf{x}$ in the topological space $X$ is $\theta^{+}$-good iff, given neighborhoods $U_{r}(r \in[\theta]<\omega)$ of $\mathbf{x}$, there are neighborhoods $V_{\alpha}(\alpha<\theta)$ of $\mathbf{x}$ such that $V_{\square} \subseteq U_{r}$ for each non-empty $r \in[\theta]^{<\omega}$.

$V_{\text {Ø }}$ will play no role in our arguments, but it would be natural to let it be $X$ when we are discussing subsets of $X$. 
Definition 2.3. A point $\mathbf{x}$ in the space $X$ is $\theta^{+}$-mediocre iff, for some fixed collection of one-to-one functions $\varphi_{\beta}: \beta \rightarrow \theta\left(\beta<\theta^{+}\right)$: whenever $U_{\xi}(\xi<\theta)$ are neighborhoods of $\mathbf{x}$, there are neighborhoods $V_{\alpha}\left(\alpha<\theta^{+}\right)$of $\mathbf{x}$ such that $V_{\alpha} \cap V_{\beta} \subseteq U_{\varphi_{\beta}(\alpha)}$ whenever $\alpha<\beta<\theta^{+}$.

Every $\omega_{1}$-mediocre point is $\omega_{1}$-OK (in the sense of [6]), but past $\omega_{1}$, the notions of "mediocre" and "OK" diverge. The arguments in this paper do not depend on which collection of functions, $\left\{\varphi_{\beta}: \beta<\theta^{+}\right\}$, is used, and we do not know how the topological properties of the mediocre points varies if this collection is varied.

Lemma 2.4. Every $\lambda^{++}$-good point is $\lambda^{+}$-mediocre.

Proof. Fix a collection of one-to-one functions $\varphi_{\beta}: \beta \rightarrow \lambda\left(\beta<\lambda^{+}\right)$. Let $U_{\xi}(\xi \in \lambda)$ be any neighborhoods of the point $\mathbf{x}$. Now define new neighborhoods $U_{r}^{*}(r \in$ $\left.\left[\lambda^{+}\right]^{<\omega}\right)$ of $\mathbf{x}$ by setting $U_{\{\alpha, \beta\}}^{*}=U_{\varphi_{\beta}(\alpha)}$ whenever $\alpha<\beta<\lambda^{+}$, and setting all other $U_{r}^{*}=X$. Apply $\lambda^{++}$-goodness to get $V_{\alpha}\left(\alpha<\lambda^{+}\right)$. Then $V_{\alpha} \cap V_{\beta} \subseteq U_{\{\alpha, \beta\}}^{*}=$ $U_{\varphi_{\beta}(\alpha)}$.

It is a folklore result that every $\kappa^{+}$-good point in a $T_{1}$ space is a weak $P_{\kappa}$-point (see, e.g., Dow 2]). Examining that proof, we see that in fact mediocrity suffices:

Lemma 2.5. If $\mathbf{x} \in X$ is a weak $P_{\kappa}$-point which is also $\kappa^{+}$-mediocre, then $\mathbf{x}$ is a weak $P_{\kappa^{+}-\text {point. }}$

Proof. Let $\varphi_{\beta}: \beta \rightarrow \kappa\left(\beta<\kappa^{+}\right)$be as in Definition 2.3. Given $Y=\left\{y_{\xi}: \xi \in \kappa\right\} \subseteq$ $X \backslash\{\mathbf{x}\}$, we wish to show that $\mathbf{x}$ is not in the closure of $Y$. For $\xi \in \kappa$, let $U_{\xi}$ be a neighborhood of $\mathbf{x}$ which misses $\left\{y_{\eta}: \eta \leq \xi\right\}$. Now, fix neighborhoods $V_{\alpha}\left(\alpha \in \kappa^{+}\right)$ of $\mathbf{x}$ such that $V_{\alpha} \cap V_{\beta} \subseteq U_{\varphi_{\beta}(\alpha)}$.

Then for some $\alpha, V_{\alpha} \cap Y=\emptyset$ : If not, then we can find $\xi \in \kappa$ and $E \subset \kappa^{+}$ with $|E|=\kappa^{+}$such that $\forall \alpha \in E\left(y_{\xi} \in V_{\alpha}\right)$. Let $\beta$ be any element of $E$ such that $|E \cap \beta|=\kappa$. So, $\varphi_{\beta}(E \cap \beta)$ is unbounded in $\kappa$. Choose $\alpha \in E \cap \beta$ such that $\varphi_{\beta}(\alpha)>\xi$. Then $V_{\alpha} \cap V_{\beta} \subseteq U_{\varphi_{\beta}(\alpha)}$ which misses $\left\{y_{\eta}: \eta \leq \varphi_{\beta}(\alpha)\right\}$. So $y_{\xi} \notin V_{\alpha} \cap V_{\beta}$, but this is a contradiction, since $\alpha, \beta \in E$.

Lemma 2.6. If $\mathbf{x}$ is $\kappa^{+}$-good in the $T_{1}$ space $X$, then $\mathbf{x}$ is a weak $P_{\kappa}$-point in $X$.

Proof. Observe that for all $\lambda<\kappa: \mathbf{x}$ is $\lambda^{++}$-good, and hence (by Lemma 2.4) $\lambda^{+}$mediocre. Now, use Lemma 2.5 and show, by induction on $\lambda<\kappa$, that $\mathbf{x}$ is a weak $P_{\lambda^{+}}$-point for all $\lambda<\kappa$.

Clearly, if $\mathbf{x} \in X \subseteq Y$ and $\mathbf{x}$ is $\kappa^{+}$-good in $Y$, then $\mathbf{x}$ is $\kappa^{+}$-good in $X$; and there are trivial examples to show that the converse is false. However,

Lemma 2.7. If $\kappa$ is regular and $\mathbf{x} \in u(\kappa)$ is $\kappa^{+}$-good in $u(\kappa)$, then $\mathbf{x}$ is $\kappa^{+}$-good in $\beta \kappa$.

Proof. Viewing $\mathbf{x}$ as an ultrafilter on $\kappa$, we prove $\kappa^{+}$-goodness in $\beta \kappa$ by fixing $U_{r} \in \mathbf{x}$ for $r \in[\kappa]^{<\omega}$ and producing $V_{\xi} \in \mathbf{x}$ for $\xi<\kappa$ such that each $V_{r} \subseteq U_{r}$. Now, $\kappa^{+}$-goodness in $u(\kappa)$ only gives us $W_{\xi} \in \mathbf{x}$ such that each $W_{\square} \subseteq^{*} U_{r}$. So, define $V_{\xi}=W_{\xi} \backslash \bigcup\left\{W_{\square} \backslash U_{r}: \max (r)=\xi\right\}$. Then each $V_{\square} \subseteq U_{r}$. Each $V_{\xi}={ }^{*} W_{\xi}$ (since $\kappa$ is regular), so each $V_{\xi} \in \mathbf{x}$ (since $\mathbf{x}$ is uniform).

We shall prove Theorem 1.2 by producing an $\mathrm{x} \in u(\kappa)$ which is both $\kappa^{+}$-good and $\kappa^{+}$-mediocre in $u(\kappa)$ (and then applying Lemmas [2.6] and [2.5), but then $\mathbf{x}$ will also be $\kappa^{+}$-good in $\beta \kappa$ (the usual sense of "good" in model theory). An obvious 
improvement of this result would be an ultrafilter which is $\kappa^{++}$-good in $u(\kappa)$, but one cannot produce that in $Z F C$ :

Theorem 2.8. If $\kappa$ is regular, $2^{\kappa}=\kappa^{+}$, and $\kappa>\omega$, then no countably incomplete $\mathbf{x} \in u(\kappa)$ is $\kappa^{++}$-good in $u(\kappa)$.

Proof. Choose $A_{n}^{\alpha} \in \mathrm{x}$ for $n<\omega \leq \alpha<\kappa^{+}$so that every $\omega$-sequence of elements of $\mathrm{x}$ is of the form $\left\langle A_{n}^{\alpha} \in \mathbf{x}: n<\omega\right\rangle$ for some $\alpha$. Then choose $B_{n}^{\alpha} \in \mathbf{x}$ so that $B_{n}^{\alpha} \subseteq A_{n}^{\alpha}$, $\bigcap_{n} B_{n}^{\alpha}=\emptyset$, and $B_{0}^{\alpha} \supseteq B_{1}^{\alpha} \supseteq B_{2}^{\alpha} \supseteq \cdots$. If $\mathbf{x}$ were $\kappa^{++}$-good in $u(\kappa)$, we could choose $D_{\alpha} \in \mathbf{x}$ for $\alpha<\kappa^{+}$so that $D_{\alpha} \cap D_{n} \subseteq^{*} B_{n+1}^{\alpha}$ whenever $n<\omega \leq \alpha<\kappa^{+}$. Now, fix $\alpha \geq \omega$ such that each $D_{n}=A_{n}^{\alpha}$. Then each $D_{\alpha} \cap B_{n}^{\alpha} \subseteq D_{\alpha} \cap A_{n}^{\alpha}=D_{\alpha} \cap D_{n} \subseteq^{*} B_{n+1}^{\alpha}$, so $D_{\alpha} \perp B_{n}^{\alpha} \backslash B_{n+1}^{\alpha}$, and hence $D_{\alpha} \perp \bigcup_{n}\left(B_{n}^{\alpha} \backslash B_{n+1}^{\alpha}\right)=B_{0}^{\alpha}$, a contradiction, since $D_{\alpha}, B_{0}^{\alpha} \in \mathbf{x}$.

Note that this theorem fails for $\kappa=\omega$, since every $P$-point is $\omega_{2}$-good. Likewise, if $\kappa$ is measurable, then every normal ultrafilter on $\kappa$ is a $P_{\kappa^{+}}$-point, and hence $\kappa^{++}$-good, in $u(\kappa)$. Furthermore, if $\kappa$ is regular and $2^{\kappa}=2^{\left(\kappa^{+}\right)}$, then there is a $\kappa^{++}$-good point in $u(\kappa)$; see [1].

\section{MATRICES}

Ultrafilters on $\kappa$ are often constructed with the aid of a matrix of sets consisting of $2^{\kappa}$ independent rows, each row being an instance of some base matrix, $\mathcal{M}$. Since $\mathcal{M}$ is sometimes a bit complicated, it may help to give an abstract discussion of such matrices:

Definition 3.1. An abstract matrix is a triple, $\mathcal{M}=(\mathcal{B}, \mathcal{J}, \mathcal{P})$, such that $\mathcal{B}$ is a boolean algebra, $\mathcal{J}$ is an ideal in $\mathcal{B}$, and $\mathcal{P} \subseteq \mathcal{B} \backslash \mathcal{J}$. A $\theta \times \mathcal{M}$ independent matrix in a boolean algebra $\mathcal{A}$ is a sequence $\left\langle h_{\alpha}: \alpha<\theta\right\rangle$, where each $h_{\alpha}: \mathcal{B} \rightarrow \mathcal{A}$ is a homomorphism, $h_{\alpha}(b)=0$ whenever $b \in \mathcal{J}$, and $\bigwedge_{\ell<n} h_{\alpha_{\ell}}\left(b_{\ell}\right) \neq 0$ whenever $n \in \omega$, each $b_{0}, \ldots, b_{n} \in \mathcal{P}$, and the $\alpha_{0}, \ldots, \alpha_{n}<\theta$ are distinct.

Of course, one can always reduce this to the case $\mathcal{J}=\{0\}$ by replacing $\mathcal{B}$ by $\mathcal{B} / \mathcal{J}$, but the definition as stated is closer to the way matrices are specified in practice, where it is simpler to take $\mathcal{B}$ to be a free algebra. For example, say we want to specify $\lambda$ disjoint elements. Let $\mathcal{B}$ be the free algebra generated by $\left\{b_{\alpha}: \alpha<\lambda\right\}$. Let $\mathcal{J}$ be generated by all $b_{\alpha} \wedge b_{\beta}$ for $\alpha<\beta<\lambda$. Let $\mathcal{P}$ (the positive elements) be $\left\{b_{\alpha}: \alpha<\lambda\right\}$. Then a $\theta \times \mathcal{M}$ independent matrix in the boolean algebra $\mathcal{P}(\kappa) /<\kappa$ consists of $\theta$ independent copies of $\lambda$ almost disjoint sets. With this $\mathcal{M}$, taking $\lambda=\kappa^{+}$and $\kappa$ regular, Dow [2] showed that there is a $2^{\kappa} \times \mathcal{M}$ independent matrix in $\mathcal{P}(\kappa) /<\kappa$. Generalizing this, we show, for any $\mathcal{M}$, how to construct a $2^{\kappa} \times \mathcal{M}$ matrix if we are given $m \times \mathcal{M}$ matrices for each $m \in \omega$.

Definition 3.2. $L=L_{\kappa}=\{\gamma<\kappa: \gamma=0$ or $\gamma$ is a limit $\} . \mathcal{F} \subseteq \omega^{\kappa}$ is mildly independent iff $f(\gamma+m) \leq m$ for all $\gamma \in L, m \in \omega, f \in \mathcal{F}$, and whenever $n<\omega$ and $f_{0}, \ldots, f_{n} \in \mathcal{F}$ are all distinct, there is a $\xi<\kappa$ such that $f_{0}(\xi), \ldots, f_{n}(\xi)$ are all distinct.

Lemma 3.3. For any infinite $\kappa$, there is a mildly independent $\mathcal{F} \subseteq \omega^{\kappa}$ with $|\mathcal{F}|=$ $2^{\kappa}$.

Proof. Fix $\mathcal{G} \subseteq \omega^{\kappa}$ which is independent in the usual sense with $|\mathcal{G}|=2^{\kappa}$ (Engelking and Karłowicz [3]). So, for all distinct $g_{0}, \ldots, g_{n} \in \mathcal{G}$ and all $i_{0}, \ldots, i_{n} \in \omega$, there are $\kappa$ different $\alpha<\kappa$ such that $g_{\ell}(\alpha)=i_{\ell}$ for $\ell=0, \ldots, n$. 
If $\kappa>\omega$, then fix $g \in \mathcal{G}$. Each $\left|g^{-1}\{m\}\right|=\kappa=|L|$, so by permuting the index set $\kappa$, we may assume that $g(\gamma+m)=m$ for all $\gamma \in L$ and $m \in \omega$. Now, let $\mathcal{F}=\{f \wedge g: f \in \mathcal{G} \backslash\{g\}\}$, where $(f \wedge g)(\xi)=\min (f(\xi), g(\xi))$.

If $\kappa=\omega$, let $I(m)=m$ for each $m$, and let $\mathcal{F}=\{f \wedge I: f \in \mathcal{G}\}$.

Theorem 3.4. Assume that $\kappa$ is regular and uncountable and let $\mathcal{M}=(\mathcal{B}, \mathcal{J}, \mathcal{P})$ be an abstract matrix. Assume that for each $m \in \omega$, there is an $m \times \mathcal{M}$ independent matrix in $\mathcal{P}(\kappa) /<\kappa$. Then there is a $2^{\kappa} \times \mathcal{M}$ independent matrix in $\mathcal{P}(\kappa) /<\kappa$.

Proof. To simplify the notation, assume that $\mathcal{J}=\{0\}$ (replace $\mathcal{B}$ by $\mathcal{B} / \mathcal{J}$ ).

For each $m$, let $\left\langle h_{j}^{m}: j \leq m\right\rangle$ be an $(m+1) \times \mathcal{M}$ independent matrix. Choose $H_{j}^{m}: \mathcal{B} \rightarrow \mathcal{P}(\kappa)$ so that $h_{j}^{m}(b)=\left[H_{j}^{m}(b)\right]$ and $H_{j}^{m}\left(b^{\prime}\right)=\kappa \backslash H_{j}^{m}(b)$; these $H_{j}^{m}$ need not be homomorphisms. Let $\mathcal{F} \subseteq \omega^{\kappa}$ be mildly independent with $|\mathcal{F}|=2^{\kappa}$. Let $D=\{(\xi, \eta): \xi \leq \eta<\kappa\}$. Then $|D|=\kappa$. Define $k_{f}: \mathcal{B} \rightarrow \mathcal{P}(D) /<\kappa$ by

$$
k_{f}(b)=\left[D \cap \bigcup\left\{\{\gamma+m\} \times H_{f(\gamma+m)}^{m}(b): \gamma \in L, m \in \omega\right\}\right] .
$$

Fix $f$; we shall show that $k_{f}$ is a homomorphism. It preserves complements by our choice of the $H_{j}^{m}$, so fix $b_{1}, b_{2}, b_{3} \in \mathcal{B}$ with $b_{3}=b_{1} \wedge b_{2}$. For each $m$ and $j, H_{j}^{m}\left(b_{3}\right)={ }^{*} H_{j}^{m}\left(b_{1}\right) \cap H_{j}^{m}\left(b_{2}\right)$, so since $\kappa>\omega$, there is a fixed $\zeta<\kappa$ such that $H_{j}^{m}\left(b_{3}\right) \Delta\left(H_{j}^{m}\left(b_{1}\right) \cap H_{j}^{m}\left(b_{2}\right)\right) \subseteq \zeta$ for every $m, j$. It follows that in $\mathcal{P}(D) /<\kappa$, $k_{f}\left(b_{3}\right) \Delta\left(k_{f}\left(b_{1}\right) \wedge k_{f}\left(b_{2}\right)\right) \leq[D \cap(\kappa \times \zeta)]=0$.

To prove independence of the $k_{f}$, fix non-zero $b_{0}, \ldots, b_{n} \in \mathcal{P}$ and distinct $f_{0}, \ldots, f_{n} \in \mathcal{F}$. By mild independence of $\mathcal{F}$, fix $\gamma \in L$ and $m \in \omega$ such that $f_{0}(\gamma+m), \ldots, f_{n}(\gamma+m)$ are all distinct. Then $\bigwedge_{\ell \leq n} k_{f_{\ell}}\left(b_{\ell}\right) \geq[\{\gamma+m\} \times$ $\left.\bigcap_{\ell \leq n} H_{f_{\ell}(\gamma+m)}^{m}\left(b_{\ell}\right)\right]>0$.

We do not know whether this theorem holds for $\kappa=\omega$, although it does hold in the case that $|\mathcal{B}| \leq \omega_{1}$, in which case one can drop the hypothesis on the $m \times \mathcal{M}$ independent matrices. First:

Lemma 3.5. Suppose that $|\mathcal{B}| \leq \omega_{1}$ and $h_{j}$ is a homomorphism from $\mathcal{B}$ into $\mathcal{P}(\omega) /$ fin for each $j<\omega$. Then one can choose $H_{j}: \mathcal{B} \rightarrow \mathcal{P}(\omega)$ such that each $h_{j}(b)=\left[H_{j}(b)\right]$, each $H_{j}\left(b^{\prime}\right)=\omega \backslash H_{j}(b)$, and for each finite subalgebra $\mathcal{A} \subseteq \mathcal{B}$, $H_{j}\lceil\mathcal{A}$ is a homomorphism from $\mathcal{A}$ into $\mathcal{P}(\omega)$ for all but finitely many $j$.

Proof. Write $\mathcal{B}$ as a continuous increasing union of countable boolean algebras, $\mathcal{B}=\bigcup_{\alpha<\omega_{1}} \mathcal{B}_{\alpha}$. By induction on $\alpha$, define all the $H_{j}\left\lceil\mathcal{B}_{\alpha}\right.$. Let $\mathcal{B}_{0}=\{0,1\}$, and let $H_{j}(0)=\emptyset$ and $H_{j}(1)=\omega$. Since there is no problem at limits, we can fix $\alpha$ and explain how to extend the $H_{j}$ to $\mathcal{B}_{\alpha+1}$. Now, write $\mathcal{B}_{\alpha+1}$ as an increasing union of finite boolean algebras, $\mathcal{B}_{\alpha+1}=\bigcup_{n<\omega} \mathcal{A}_{n}$, with $\mathcal{A}_{0}=\{0,1\}$. We may assume that we have $n_{0} \leq n_{1} \leq n_{2} \leq \cdots$ with $\sup _{j} n_{j}=\omega$ and each $H_{j} \uparrow\left(\mathcal{A}_{n_{j}} \cap \mathcal{B}_{\alpha}\right)$ a homomorphism. Since $\mathcal{A}_{n_{j}}$ is finite, one can extend $H_{j} \uparrow\left(\mathcal{A}_{n_{j}} \cap \mathcal{B}_{\alpha}\right)$ to $\mathcal{A}_{n_{j}}$ so that it is still a homomorphism. Now that we have $H_{j}$ defined on $\mathcal{B}_{\alpha} \cup \mathcal{A}_{n_{j}}$, we can extend it to all of $\mathcal{B}_{\alpha+1}$ by the Axiom of Choice.

Theorem 3.6. Let $\mathcal{M}=(\mathcal{B}, \mathcal{J}, \mathcal{P})$ be an abstract matrix, with $|\mathcal{B}| \leq \omega_{1}$. Then there is a $2^{\omega} \times \mathcal{M}$ independent matrix in $\mathcal{P}(\omega) /$ fin.

Proof. Let the $h_{j}^{m}: \mathcal{B} \rightarrow \mathcal{P}(\omega) /$ fin be as in the proof of Theorem 3.4. This is possible because every boolean algebra of size $\omega_{1}$ (in particular, the direct sum of $m$ copies of $\mathcal{B} / \mathcal{J})$ can be embedded into $\mathcal{P}(\omega) /$ fin. 
Now, follow the proof of Theorem [3.4 but choose the $H_{j}^{m}: \mathcal{B} \rightarrow \mathcal{P}(\kappa)$ as in Lemma 3.5, so that whenever $b_{3}=b_{1} \wedge b_{2}$, we have $H_{j}^{m}\left(b_{3}\right)=H_{j}^{m}\left(b_{1}\right) \cap H_{j}^{m}\left(b_{2}\right)$ for all but finitely many $m, j$. Define the $k_{f}$ in precisely the same way, although $D$ can also be $\omega \times \omega$ now.

\section{The Hat Trick}

Definition 4.1. A hat function is a function $\widehat{-}:\left[\kappa^{+}\right]^{<\omega} \rightarrow[\kappa]^{<\omega}$ satisfying:

- $p \subseteq q \Rightarrow \widehat{p} \subseteq \widehat{q}$.

- $\widehat{\emptyset}=\emptyset$.

If $\varphi_{\beta}: \beta \rightarrow \kappa$ is one-to-one for each $\beta<\kappa^{+}$, the derived hat function is defined by $\widehat{p}=\left\{\varphi_{\beta}(\alpha): \alpha, \beta \in p \wedge \alpha<\beta\right\}$.

We have three goals in introducing this notion: First, to simplify the the construction of a mediocre point by referring to rather than to the sequence $\left\langle\varphi_{\beta}: \beta<\kappa^{+}\right\rangle$. Second (Lemma 4.10), to get an $m \times \mathcal{M}$ independent matrix immediately from a $1 \times \mathcal{M}$ independent matrix. Third (Section 5), to unify the notions of "good", "mediocre", and "OK" into one kind of point.

Definition 4.2. A step-family (over $\kappa$, with respect to $\widehat{-}$ ) is an indexed collection of subsets of $\kappa,\left\{E_{s}: s \in[\kappa]^{<\omega}\right\} \cup\left\{A_{\alpha}: \alpha<\kappa^{+}\right\}$, satisfying:

S1. $E_{s} \cap E_{t}=\emptyset$ for each distinct $s, t \in[\kappa]^{<\omega}$.

S2. $A_{\square} \perp \bigcup\left\{E_{s}: s \nsupseteq \widehat{p}\right\}$ for each $p \in\left[\kappa^{+}\right]^{<\omega}$.

S3. $\widehat{p} \subseteq s \Rightarrow\left|A_{[p} \cap E_{s}\right|=\kappa$ for each $p \in\left[\kappa^{+}\right]^{<\omega}$ and $s \in[\kappa]^{<\omega}$.

Definition 4.3. If $\mathcal{F}$ is any filter on $\kappa$, then $\mathcal{F}^{+}=\{X \subseteq \kappa: \kappa \backslash X \notin \mathcal{F}\}$. $\mathcal{F} \mathcal{R}=$ $\mathcal{F} \mathcal{R}(\kappa)$ is the Fréchet filter, $\{X \subseteq \kappa:|\kappa \backslash X|<\kappa\}$.

So, $\mathcal{F} \mathcal{R}^{+}=[\kappa]^{\kappa}$.

Definition 4.4. Given any index set $I$ and filter $\mathcal{F}$ on $\kappa$, the indexed collection $\left\{E_{s}^{i}: s \in[\kappa]^{<\omega}, i \in I\right\} \cup\left\{A_{\alpha}^{i}: \alpha<\kappa^{+}, i \in I\right\}$ is an independent matrix of $|I|$ step-families (over $\kappa$ ) with respect to $\mathcal{F}$, $\widehat{\text { iff: }}$

1. For each fixed $i \in I,\left\{E_{s}^{i}: s \in[\kappa]^{<\omega}\right\} \cup\left\{A_{\alpha}^{i}: \alpha<\kappa^{+}\right\}$is a step-family.

2. Given $n \in \omega, p_{0}, p_{1}, \ldots, p_{n-1} \in\left[\kappa^{+}\right]^{<\omega}, s_{0}, s_{1}, \ldots, s_{n-1} \in[\kappa]^{<\omega}$, and distinct $i_{0}, i_{1}, \ldots, i_{n-1} \in I$, if each $\widehat{p}_{\ell} \subseteq s_{\ell}$ then

$$
\left(A_{\left[p_{0}\right.}^{i_{0}} \cap E_{s_{0}}^{i_{0}}\right) \cap\left(A_{\left[p_{1}\right.}^{i_{1}} \cap E_{s_{1}}^{i_{1}}\right) \cap \cdots \cap\left(A_{\left[p_{n-1}\right.}^{i_{n-1}} \cap E_{s_{n-1}}^{i_{n-1}}\right) \in \mathcal{F}^{+} .
$$

Theorem 4.5. If $\kappa$ is regular and $\widehat{-}$ is any hat function, then there is an independent matrix of $2^{\kappa}$ step-families over $\kappa$ with respect to $\mathcal{F R}(\kappa), \widehat{ }$.

The rest of this section is devoted to proving this theorem, which will get used in the construction in Section 6 So, - will be fixed and the filter $\mathcal{F}$ will be understood to be $\mathcal{F} \mathcal{R}$. We also assume that $\kappa$ is regular, in which case the following lemma is easily proved:

Lemma 4.6. Given $A_{\xi} \subseteq \kappa$ for $\xi<\kappa$, there are sets $B_{\xi} \subseteq \kappa$ such that:

- $A_{\xi}={ }^{*} B_{\xi}$.

- $A_{\xi} \subseteq^{*} A_{\eta} \Rightarrow B_{\xi} \subseteq B_{\eta}$.

- $A_{\xi} \perp A_{\eta} \Rightarrow B_{\xi} \cap B_{\eta}=\emptyset$. 
Corollary $4.7\left((\kappa, \kappa)\right.$ separation property). Given subsets $S_{\xi}(\xi<\kappa)$ and $T_{\eta}(\eta<$ $\kappa)$ of $\kappa$ such that each $S_{\xi} \perp T_{\eta}$, there is some $W \subseteq \kappa$ such that $\forall \xi\left(W \supseteq{ }^{*} S_{\xi}\right)$ and $\forall \eta\left(W \perp T_{\eta}\right)$.

Proof. By Lemma 4.6 we can assume that actually each $S_{\xi} \cap T_{\eta}=\emptyset$, and then we can set $W=\bigcup_{\xi} S_{\xi}$.

The following lemma does most of the work involved in getting a single stepfamily:

Lemma 4.8. Assume that $Q$ is a subset of $\left[\kappa^{+}\right]^{<\omega}$ which is closed downward and which contains $\emptyset$. Then there are $X_{\alpha} \subseteq \kappa$ for $\alpha<\kappa^{+}$such that for all $p \in\left[\kappa^{+}\right]^{<\omega}$, $\left|X_{\square}\right|=\kappa \Longleftrightarrow p \in Q$.

Proof. Let $\left(\kappa^{+}\right)^{<\omega \uparrow}$ denote all the strictly increasing finite sequences from $\kappa^{+}$. Choose $Y_{\sigma}, Z_{\alpha}^{\sigma} \in[\kappa]^{\kappa}$ (for $\sigma \in\left(\kappa^{+}\right)^{<\omega \uparrow}, \max \sigma<\alpha<\kappa^{+}$) so that $Y_{()}=\kappa$, and for each $\sigma$ :

- $Z_{\beta}^{\sigma} \subseteq Y_{\sigma}$ whenever $\max \sigma<\beta<\kappa^{+}$.

- $Z_{\beta}^{\sigma} \varsubsetneqq Z_{\alpha}^{\sigma}$ whenever $\max \sigma<\alpha<\beta<\kappa^{+}$.

- $Y_{\sigma \frown \alpha}=Z_{\alpha}^{\sigma} \backslash Z_{\alpha+1}^{\sigma}$.

Then note that $Y_{\sigma \frown \alpha} \subseteq Y_{\sigma}$, and $\alpha \neq \beta \Rightarrow Y_{\sigma-\alpha} \perp Y_{\sigma \frown \beta}$. So, the $Y_{\sigma}$ form a tree of subsets of $\kappa$ with $\operatorname{root} Y_{()}=\kappa$. Each node $Y_{\sigma}$ has $\kappa^{+}$almost disjoint children, $Y_{\sigma-\alpha}\left(\max \sigma<\alpha<\kappa^{+}\right)$. The $Z_{\beta}^{\sigma}$ let us achieve an instance of $\left(\kappa, \kappa^{+}\right)$separation (see (5) below), when all we have is $(\kappa, \kappa)$-separation.

Now, we choose the sets $X_{\beta}$ by induction on $\beta$. Let $\Sigma_{\beta}=\left\{\sigma \in\left(\kappa^{+}\right)^{<\omega \uparrow}\right.$ : $\max \sigma=\beta \wedge \operatorname{ran} \sigma \in Q\}$. Using $(\kappa, \kappa)$-separation, choose $X_{\beta}$ so that:

(1) $\tau \in \Sigma_{\beta} \Rightarrow X_{\beta} \supseteq^{*} Y_{\tau}$.

(2) $\left(p \in[\beta]^{<\omega} \wedge p \cup\{\beta\} \notin Q\right) \Rightarrow X_{\beta} \perp X_{[p}$.

(3) $\sigma \in(\beta)^{<\omega \uparrow} \Rightarrow X_{\beta} \perp Z_{\beta+1}^{\sigma}$.

(4) $\left(\sigma \in(\beta)^{<\omega \uparrow} \wedge \sigma^{\frown} \beta \notin \Sigma_{\beta}\right) \Rightarrow X_{\beta} \perp Z_{\beta}^{\sigma}$.

Only (1) and (2) are needed to show that the $X_{\alpha}$ satisfy the lemma, but (3) and (4) are necessary in order to continue the induction. (3) implies

(5) $\beta \notin \operatorname{ran} \tau \wedge \beta<\max \tau \Rightarrow X_{\beta} \perp Y_{\tau}$.

To see this, write $\tau=\sigma^{\frown} \gamma^{\frown} \rho$ where $\sigma \in(\beta)^{<\omega \uparrow}, \gamma>\beta$, and $\rho$ fills out the rest of $\tau$. Then $Y_{\tau} \subseteq Y_{\sigma-\gamma} \subseteq Z_{\gamma}^{\sigma} \subseteq^{*} Z_{\beta+1}^{\sigma}$, so apply (3).

In order to see that $(\kappa, \kappa)$-separation really applies to get $X_{\beta}$ as above, we need to check that the $Y_{\tau}$ from (1) are almost disjoint from the $X_{[p}, Z_{\beta+1}^{\sigma}, Z_{\beta}^{\sigma}$ from $(2,3,4)$. Fix $\tau=\pi \frown \beta \in \Sigma_{\beta}$, and note that by the definition of $\Sigma_{\beta}$, we have $\operatorname{ran}\left(\pi^{\frown} \beta\right) \in Q$.

For (1) with (2), fix $p \in[\beta]^{<\omega}$ such that $p \cup\{\beta\} \notin Q$. Since $Q$ is closed downward, this implies that $\exists \alpha \in p$ such that $\alpha \notin \operatorname{ran} \pi$. By (5) applied inductively to $\alpha, X_{\alpha} \perp Y_{\tau}$ and therefore $X_{[} \perp Y_{\tau}$.

For (1) with (3) and (4), fix $\sigma \in(\beta)^{<\omega \uparrow}$. Since $\tau=\pi \frown \beta$ cannot be an initial sequence of $\sigma$, there are three cases to consider.

Case 1: Neither of $\sigma$ and $\tau$ is an initial sequence of the other: Then $Z_{\beta+1}^{\sigma} \subseteq^{*}$ $Z_{\beta}^{\sigma} \subseteq Y_{\sigma} \perp Y_{\tau}$.

Case 2: $\sigma=\pi$ : Then (4) cannot happen, and $Y_{\tau}=Y_{\sigma \frown \beta} \perp Z_{\beta+1}^{\sigma}$.

Case 3: $\sigma$ is a proper initial sequence of $\pi$. Say $\pi=\sigma^{\frown} \alpha \frown \rho$, where $\alpha<\beta$. Then $Y_{\tau} \subseteq Y_{\sigma-\alpha}=Z_{\alpha}^{\sigma} \backslash Z_{\alpha+1}^{\sigma} \perp Z_{\beta}^{\sigma}$. 
We now prove: $\left|X_{[q}\right|=\kappa \Longleftrightarrow q \in Q$. Fix $q \in\left[\kappa^{+}\right]^{<\omega}$. If $q \notin Q$, then write $q=p \cup\{\beta\}$ where $\beta$ is the maximum element of $q$ and $p \in[\beta]^{<\omega}$; then (2) implies $\left|X_{[q}\right|=\left|X_{\beta} \cap X_{[p}\right|<\kappa$. If $q \in Q$, write $q=\left\{\alpha_{0}, \alpha_{1}, \ldots, \alpha_{n}\right\}$ where $\alpha_{0}<\alpha_{1}<\cdots<\alpha_{n}$. Let $\sigma=\left(\alpha_{0}, \alpha_{1}, \ldots, \alpha_{n}\right)$. Then each $\left(\alpha_{0}, \alpha_{1}, \ldots, \alpha_{i}\right) \in \Sigma_{\alpha_{i}}$, so $Y_{\sigma} \subseteq Y_{\left(\alpha_{0} \cdots \alpha_{i}\right)} \subseteq^{*} X_{\alpha_{i}}$ by (1). Therefore $Y_{\sigma} \subseteq^{*} X_{\square}$, so $\left|X_{\square}\right|=\kappa$.

Lemma 4.9. Given any hat function - , there exists a step-family, with respect to $\widehat{-},\left\{E_{s}: s \in[\kappa]^{<\omega}\right\} \cup\left\{A_{\alpha}: \alpha<\kappa^{+}\right\}$.

Proof. Let $\bigcup\left\{E_{s}: s \in[\kappa]^{<\omega}\right\}=\kappa$ be any partition of $\kappa$ into sets of size $\kappa$. For $\beta<\kappa^{+}$, we define $A_{\beta}$ roughly by defining each $A_{\beta} \cap E_{s}$.

For $s \in[\kappa]^{<\omega}$, set $Q_{s}=\left\{p \in\left[\kappa^{+}\right]^{<\omega}: \widehat{p} \subseteq s\right\}$. Then $Q_{s}$ is as in Lemma 4.8, so we can choose subsets $X_{\alpha}^{s}$ of $E_{s}$ such that $\left|X_{[p}^{s}\right|=\kappa \Longleftrightarrow \widehat{p} \subseteq s$.

As a first approximation to the $A_{\beta}$, define $C_{\beta}=\bigcup\left\{X_{\beta}^{s}: s \in[\kappa]^{<\omega}\right\}$. Then $C_{\beta} \cap E_{s}=X_{\beta}^{s}$ so $\left|C_{p} \cap E_{s}\right|=\kappa \Longleftrightarrow \widehat{p} \subseteq s$. These $C_{\beta}$ satisfy part (S3) of Definition 4.2, but they might fail to satisfy (S2). We have $\left|C_{[p} \cap E_{s}\right|<\kappa$ whenever $s \nsupseteq \widehat{p}$, but we need $\left|C_{[p} \cap \bigcup\left\{E_{s}: s \nsupseteq \widehat{p}\right\}\right|<\kappa$.

Now, choose $A_{\beta}$ (by induction on $\beta<\kappa^{+}$) such that:

(i) $A_{\beta} \supseteq^{*} C_{\beta} \cap E_{s}$ for all $s \in[\kappa]^{<\omega}$.

(ii) $A_{\beta} \perp E_{s} \backslash C_{\beta}$ for all $s \in[\kappa]^{<\omega}$.

(iii) $\left.A_{\beta} \perp \bigcup\left\{A_{p} \cap E_{t}: t \nsupseteq p \widehat{\cup\{\beta}\right\}\right\}$ for all $p \in[\beta]^{<\omega}$.

Assuming this can be done, (i) and (ii) give us also

(iv) $A_{p} \cap E_{s}={ }^{*} C_{[p} \cap E_{s}$.

By (iv), the $A_{\beta}$ also satisfy (S3), and (iii) implies that the $A_{\beta}$ satisfy (S2).

To prove that such $A_{\beta}$ can be chosen, we apply $(\kappa, \kappa)$-separation, but $(\mathrm{i}, \mathrm{iii})$ requires us to verify that for all $s \in[\kappa]^{<\omega}$ and all $p \in[\beta]^{<\omega}$

$$
\left.D_{\beta, s}:=\left(C_{\beta} \cap E_{s}\right) \cap \bigcup\left\{A_{\square} \cap E_{t}: t \nsupseteq \widehat{p \cup\{\beta}\right\}\right\}={ }^{*} \emptyset .
$$

If $s \supseteq \widehat{p \cup\{\beta}$, then $D_{\beta, s}=\emptyset$, so assume that $s \nsupseteq \widehat{\cup \cup\{\beta}$. Then $D_{\beta, s}=C_{\beta} \cap E_{s} \cap$ $A_{\square}$. Applying (iv) inductively, $D_{\beta, s}={ }^{*} C_{[p \cup\{\beta\}} \cap E_{s}$, which has size less than $\kappa$.

Lemma 4.10. If $\kappa$ is regular, $\widehat{-}$ is any hat function, and $n<\omega$, then there is an independent matrix of $n$ step-families over $\kappa$ with respect to $\mathcal{F R}(\kappa), \widehat{\text { : }}$ $\left\{E_{s}^{i}: s \in[\kappa]^{<\omega}, i<n\right\} \cup\left\{A_{\alpha}^{i}: \alpha<\kappa^{+}, i<n\right\}$.

Proof. We apply Lemma 4.9, changing the index set to $\left[n \times \kappa^{+}\right]^{<\omega}$. For $P \in$ $\left[n \times \kappa^{+}\right]^{<\omega}$ and $i<n$, let $P_{i}=\{\alpha:(i, \alpha) \in P\}$, and define

$$
\widetilde{P}=\left(\{0\} \times \widehat{P_{0}}\right) \cup\left(\{1\} \times \widehat{P_{1}}\right) \cup \cdots \cup\left(\{n-1\} \times \widehat{P_{n-1}}\right) .
$$

Then $\sim$ is a hat function from $\left[n \times \kappa^{+}\right]^{<\omega}$ to $[n \times \kappa]^{<\omega}$. So, we have a step-family with respect to $\sim,\left\{E_{S}: S \in[n \times \kappa]^{<\omega}\right\} \cup\left\{A_{(i, \alpha)}: i<n, \alpha<\kappa^{+}\right\}$.

For each $i<n$ and $s \in[\kappa]^{<\omega}$, let $E_{s}^{i}=\bigcup\left\{E_{S}: S_{i}=s\right\}$. Since all the $E_{S}$ are disjoint, the $E_{s}^{i}$, for each fixed $i$, are also disjoint. Let $A_{\alpha}^{i}=A_{(i, \alpha)}$.

Fix $i<n$ and $p \in\left[\kappa^{+}\right]^{<\omega}$; we verify condition (S2) in the definition of stepfamily; that is, $A_{[p}^{i} \perp \bigcup\left\{E_{s}^{i}: s \nsupseteq \hat{p}\right\}$. Now $A_{[\eta}^{i}=A_{[\{i\} \times p}$, and

$$
\bigcup\left\{E_{s}^{i}: s \nsupseteq \widehat{p}\right\}=\bigcup\left\{E_{S}: S \nsupseteq\{\widetilde{i\} \times p}\},\right.
$$

so we apply (S2) with respect to $\sim$. 
Observe that for $S \in[n \times \kappa]^{<\omega}$ and $P \in\left[n \times \kappa^{+}\right]^{<\omega}$, we have

$$
\begin{aligned}
E_{S} & =E_{S_{0}}^{0} \cap E_{S_{1}}^{1} \cap \cdots \cap E_{S_{n-1}}^{n-1}, \\
A_{[} & =A_{P_{0}}^{0} \cap A_{P_{1}}^{1} \cap \cdots \cap A_{P_{n-1}}^{n-1} .
\end{aligned}
$$

To prove independence (which implies (S3) for each step-family), fix $p_{\ell}, s_{\ell}$ for $\ell<n$ as in Definition 4.42 (here, $I=n$ and $i_{\ell}=\ell$ ). Assume each $\widehat{p}_{i} \subseteq s_{i}$. Define $P, S$ so that $P_{i}=p_{i}$ and $S_{i}=s_{i}$ for each $i$. Then $\widetilde{P} \subseteq S$, so $\left|A_{\mid P} \cap E_{S}\right|=\kappa$, which implies $(*)$ in Definition 4.4 .

Proof of Theorem 4.5. We apply Theorem 3.4 (or Theorem 3.6 in the case $\kappa=\omega$ ), but one must use a little care because the definition of step-family involves infinite unions of the $E_{s}$. We handle this by adding a "name", $b_{t}$, to represent $B_{t}:=\bigcup\left\{E_{s}\right.$ : $s \nsupseteq t\}$.

Let $\mathcal{B}$ be the free algebra generated by elements $a_{\alpha}$ for $\alpha<\kappa^{+}$, together with elements $e_{s}$ and $b_{s}$ for $s \in[\kappa]^{<\omega}$. Let $\mathcal{J}$ be generated by:

J1. $e_{s} \wedge e_{t}$ whenever $s, t$ are distinct.

J2. $e_{s} \backslash b_{t}$ whenever $s \nsupseteq t$.

J3. $\left(\bigwedge_{\alpha \in p} a_{\alpha} \wedge b_{\widehat{p}}\right)$ for each $p \in\left[\kappa^{+}\right]^{<\omega}$.

Let $\mathcal{P}$ contain

P1. $e_{s} \wedge \bigwedge_{\alpha \in p} a_{\alpha}$ whenever $\widehat{p} \subseteq s$.

By Lemma 4.10 there is an $m \times \mathcal{M}$ independent matrix for each finite $m$. Hence, there is a $2^{\kappa} \times \mathcal{M}$ independent matrix $\left\langle h_{\alpha}: \alpha<2^{\kappa}\right\rangle$ in $\mathcal{P}(\kappa) /<\kappa$.

Now, fix $\alpha$. Since $\left|[\kappa]^{<\omega}\right|=\kappa$, which is regular, we can apply Lemma 4.6] to choose representatives $E_{s}^{\alpha}$ and $B_{t}^{\alpha}$ such that $h_{\alpha}\left(e_{s}\right)=\left[E_{s}^{\alpha}\right], h_{\alpha}\left(b_{s}\right)=\left[B_{s}^{\alpha}\right]$, the $E_{s}^{\alpha}$ are really disjoint (using (J1)), and $E_{s}^{\alpha} \subseteq B_{t}^{\alpha}$ whenever $s \nsupseteq t$ (using (J2)). Then condition (J3) ensures that $A_{p} \perp \bigcup\left\{E_{s}: s \nsupseteq \widehat{p}\right\}$. Condition (P1) now ensures that $\widehat{p} \subseteq s \Rightarrow\left|A_{p} \cap E_{s}\right|=\kappa$. 4.4 .

Thus, the matrix gives us $2^{\kappa}$ independent step-families in the sense of Definition

\section{HATPOinTS}

Using the hat functions, we see that "good" and "mediocre" are different flavors of the same notion. Throughout, $\kappa$ is a fixed regular cardinal.

Definition 5.1. Let $\widehat{\widehat{C}}:\left[\kappa^{+}\right]^{<\omega} \rightarrow[\kappa]^{<\omega}$ be a hat function and let $\mathbf{x}$ be a point in $X$. Then $\mathbf{x}$ is a point in $X$ iff, given neighborhoods $U_{r}\left(r \in[\kappa]^{<\omega}\right)$ of $\mathbf{x}$, there are neighborhoods $V_{\alpha}\left(\alpha<\kappa^{+}\right)$of $\mathbf{x}$ such that $V_{\square} \subseteq U_{\widehat{r}}$ for each non-empty $r \in\left[\kappa^{+}\right]^{<\omega}$.

For example, if $\widehat{p}=p \cap \kappa$ for all $p$, then $\mathbf{x}$ is a - point iff $\mathbf{x}$ is $\kappa^{+}$-good. In this case, the $V_{\alpha}$ for $\alpha \geq \kappa$ are irrelevant.

Lemma 5.2. There is a hat function $-\quad:\left[\kappa^{+}\right]^{<\omega} \rightarrow[\kappa]^{<\omega}$ such that every point is both $\kappa^{+}$-mediocre and $\kappa^{+}$-good.

Proof. Let $\left\langle\varphi_{\beta}: \beta<\kappa^{+}\right\rangle$be as in Definition [2.3 but assume also that $\varphi_{\beta}(\alpha)=\alpha$

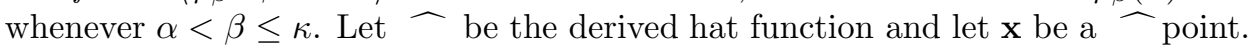
Applying Definition 5.1 with $r=\{\alpha, \beta\}$ shows that $\mathbf{x}$ is $\kappa^{+}$-mediocre. To show 
that $\mathbf{x}$ is $\kappa^{+}$-good, let $W_{\alpha}=V_{\alpha} \cap V_{\kappa}$. Then for $r \in[\kappa]<\omega, \widehat{r \cup\{\kappa\}}=r$, so $W_{\text {田 }}=V_{r r \cup\{\kappa\}} \subseteq U_{r}$

Remark 5.3. In the definition (5.1) of "hatpoint", it is sufficient to consider only monotone sequences of neigborhoods, $\left\langle U_{r}: r \in[\kappa]^{<\omega}\right\rangle$ of $\mathbf{x}$; that is $r \subseteq s \Rightarrow U_{r} \supseteq$ $U_{s}$.

Proof. Replace each $U_{r}$ by $\bigcap\left\{U_{s}: s \subseteq r\right\}$.

Keisler's original definition of "good" had "monotone", and restricting to monotone sequences will simplify the construction in Section 6 .

In the definition of hat function and hatpoint, one could more generally consider $\widehat{\widehat{C}}:[\theta]^{<\omega} \rightarrow[\kappa]^{<\omega}$. For example, fix any $\theta$, let $\kappa=\omega$, and define $\widehat{r}=\{0,1, \ldots,|r|\}$. Then the $\widehat{-}$ points are just the $\theta$-OK points from [6].

\section{Proof of Theorem 1.2}

Applying Lemmas 5.2, 2.6 and 2.5, Theorem 1.2 is immediate from the following more general result:

Theorem 6.1. If $\kappa$ is regular and $\widehat{\widehat{C}}:\left[\kappa^{+}\right]^{<\omega} \rightarrow[\kappa]^{<\omega}$ is a hat function, then there is an $\mathbf{x} \in u(\kappa)$ which is a point in $u(\kappa)$.

Proof. Apply Theorem 4.5, and fix a matrix of $2^{\kappa}$ step-families (over $\kappa$ ), $\left\{E_{s}^{i}\right.$ : $\left.s \in[\kappa]^{<\omega}, i \in 2^{\kappa}\right\} \cup\left\{A_{\alpha}^{i}: \alpha<\kappa^{+}, i \in 2^{\kappa}\right\}$, which is independent with respect to $\mathcal{F} \mathcal{R}(\kappa)$. Assume that for each $i$, we have, in addition to (S1), (S2), (S3) in Definition 4.2

S4. $\bigcup\left\{E_{s}^{i}: s \in[\kappa]^{<\omega}\right\}=\kappa$.

S5. $A_{[p}^{i} \subseteq^{*} \bigcup\left\{E_{s}^{i}: s \supseteq \widehat{p}\right\}$ for each $p \in\left[\kappa^{+}\right]^{<\omega}$.

To get (S4), first shrink the $A_{\alpha}^{i}$ so that each $A_{\alpha}^{i} \subseteq \bigcup\left\{E_{s}^{i}: s \in[\kappa]^{<\omega}\right\}$, and then expand the $E_{s}^{i}$ so that $\left\{E_{s}^{i}: s \in[\kappa]^{<\omega}\right\}$ forms a partition of $\kappa$. Then, (S5) follows from $(\mathrm{S} 2, \mathrm{~S} 4)$.

We plan to construct the ultrafilter $\mathbf{x}$ by induction, in $2^{\kappa}$ steps. We have two basic tasks: making $\mathbf{x}$ "ultra" and making $\mathbf{x}$ a point. To accomplish these, let $B_{\mu}, C_{r}^{\mu}$ be subsets of $\kappa$, for $\mu<2^{\kappa}, r \in[\kappa]^{<\omega}$, so that:

- $\mathcal{P}(\kappa)=\left\{B_{\mu}: \mu<2^{\kappa} \wedge \mu \equiv 0 \bmod 2\right\}$.

- Each $\left\langle C_{r}^{\mu}: r \in[\kappa]<\omega\right\rangle$ is monotone (see Remark [5.3), and every monotone sequence in $\mathcal{P}(\kappa)^{\left([\kappa]^{<\omega}\right)}$ appears as $\left\langle C_{r}^{\mu}: r \in[\kappa]^{<\omega}\right\rangle$ for $2^{\kappa}$ distinct $\mu$ such that $\mu \equiv 1 \bmod 2$.

We shall construct $\mathbf{x}$ as an increasing union of filters: $\mathbf{x}=\bigcup_{\mu<2^{\kappa}} \mathcal{F}_{\mu}$. Set $\mathcal{F}_{0}=\mathcal{F} \mathcal{R}(\kappa)$ and $I_{0}=2^{\kappa}$. We define $\mathcal{F}_{\mu}$ and $I_{\mu}\left(\mu<2^{\kappa}\right)$ so that:

(1) If $\mu<\nu$, then $\mathcal{F}_{\mu} \subseteq \mathcal{F}_{\nu}$ and $I_{\mu} \supseteq I_{\nu}$.

(2) For limit $\nu: \mathcal{F}_{\nu}=\bigcup_{\mu<\nu} \mathcal{F}_{\mu}$ and $I_{\nu}=\bigcap_{\mu<\nu} I_{\mu}$.

(3) Each $I_{\mu} \backslash I_{\mu+1}$ is finite.

(4) Each $\mathcal{F}_{\mu}$ is a filter on $\kappa$, and the matrix $\left\{E_{s}^{i}: s \in[\kappa]<\omega, i \in I_{\mu}\right\} \cup\left\{A_{\alpha}^{i}\right.$ : $\left.\alpha<\kappa^{+}, i \in I_{\mu}\right\}$ of remaining step-families is independent w.r.t. $\mathcal{F}_{\mu}$.

(5) If $\mu \equiv 0$, then either $B_{\mu} \in \mathcal{F}_{\mu+1}$ or $\kappa \backslash B_{\mu} \in \mathcal{F}_{\mu+1}$.

(6) If $\mu \equiv 1$ and each $C_{r}^{\mu} \in \mathcal{F}_{\mu}$, then there are $D_{\alpha}^{\mu} \in \mathcal{F}_{\mu+1}$ for $\alpha<\kappa^{+}$such that $\forall p \in\left[\kappa^{+}\right]<\omega\left[D_{[p}^{\mu} \subseteq^{*} C_{\widehat{p}}^{\mu}\right]$. 
As usual in these constructions, there is no problem at limits, so we proceed to describe the successor step. For $\mathcal{E} \subseteq \mathcal{P}(\kappa)$, let $\langle\langle\mathcal{E}\rangle\rangle$ denote the filter generated by $\mathcal{E}$.

If $\mu \equiv 0$ : If $\left\langle\left\langle\mathcal{F}_{\mu} \cup\left\{B_{\mu}\right\}\right\rangle\right\rangle$ is a proper filter and the matrix of step-families $\left\{E_{s}^{i}: s \in[\kappa]^{<\omega}, i \in I_{\mu}\right\} \cup\left\{A_{\alpha}^{i}: \alpha<\kappa^{+}, i \in I_{\mu}\right\}$ is independent w.r.t. $\left\langle\left\langle\mathcal{F}_{\mu} \cup\left\{B_{\mu}\right\}\right\rangle\right\rangle$, then set $\mathcal{F}_{\mu+1}=\left\langle\left\langle\mathcal{F}_{\mu} \cup\left\{B_{\mu}\right\}\right\rangle\right\rangle$ and $I_{\mu+1}=I_{\mu}$. Otherwise, fix $n \in \omega$, distinct $i_{\ell} \in I_{\mu}(\ell<n)$, and $\widehat{p}_{\ell} \subseteq s_{\ell}(\ell<n)$, such that

$$
B_{\mu} \cap\left(A_{\underline{p_{0}}}^{i_{0}} \cap E_{s_{0}}^{i_{0}}\right) \cap\left(A_{\underline{p_{1}}}^{i_{1}} \cap E_{s_{1}}^{i_{1}}\right) \cap \cdots \cap\left(A_{\underline{p_{n-1}}}^{i_{n-1}} \cap E_{s_{n-1}}^{i_{n-1}}\right) \notin \mathcal{F}_{\mu}^{+} .
$$

Set $\mathcal{F}_{\mu+1}=\left\langle\left\langle\mathcal{F}_{\mu} \cup\left\{A_{\left[p_{0}\right.}^{i_{0}}, \ldots, A_{\left[p_{n-1}\right.}^{i_{n-1}}\right\} \cup\left\{E_{s_{0}}^{i_{0}}, \ldots, E_{s_{n-1}}^{i_{n-1}}\right\}\right\rangle\right.$ and set $I_{\mu+1}=$ $I_{\mu} \backslash\left\{i_{0}, i_{1}, \ldots, i_{n-1}\right\}$. Then $\kappa \backslash B_{\mu} \in \mathcal{F}_{\mu+1}$, and we leave it to the reader to check that condition (4) holds for $\mathcal{F}_{\mu+1}$ and $I_{\mu+1}$.

If $\mu \equiv 1$ : Assume each $C_{s}^{\mu}\left(s \in[\kappa]^{<\omega}\right)$ is in $\mathcal{F}_{\mu}$. (Otherwise, set $\mathcal{F}_{\mu+1}=\mathcal{F}_{\mu}$ and $I_{\mu+1}=I_{\mu}$.) Choose $i \in I_{\mu}$ and set $I_{\mu+1}=I_{\mu} \backslash\{i\}$. We shall leave off the superscripts $\mu$ and $i$ when they are clear from context. For $\alpha<\kappa^{+}$, define

$$
D_{\alpha}=A_{\alpha} \cap \bigcup\left\{C_{s} \cap E_{s}: s \in[\kappa]^{<\omega}\right\} .
$$

Then for $p \in\left[\kappa^{+}\right]^{<\omega}$,

$$
\begin{aligned}
D_{\square} & =A_{[} \cap \bigcup\left\{C_{s} \cap E_{s}: s \in[\kappa]^{<\omega}\right\} \\
& \subseteq{ }^{*} \bigcup\left\{E_{s}: s \supseteq \widehat{p}\right\} \cap \bigcup\left\{C_{s} \cap E_{s}: s \in[\kappa]^{<\omega}\right\} \\
& =\bigcup\left\{C_{s} \cap E_{s}: s \supseteq \widehat{p}\right\} \\
& \subseteq \bigcup\left\{C_{s}: s \supseteq \widehat{p}\right\} \\
& =C_{\widehat{p}},
\end{aligned}
$$

by monotonicity of $\left\langle C_{p}: p \in[\kappa]^{<\omega}\right\rangle$. Set $\mathcal{F}_{\mu+1}=\left\langle\left\langle\mathcal{F}_{\mu} \cup\left\{D_{\alpha}: \alpha<\kappa^{+}\right\}\right\rangle\right\rangle$. To verify condition (4): Any element of $\mathcal{F}_{\mu+1}$ is of the form $B \cap D_{\underline{p}}$ for some $B \in \mathcal{F}_{\mu}$ and $p \in\left[\kappa^{+}\right]^{<\omega}$. But $D_{[p} \supseteq A_{[p} \cap C_{\widehat{p}} \cap E_{\widehat{p}}$, and $C_{\widehat{p}} \in \mathcal{F}_{\mu}$, so condition (4) for $\mathcal{F}_{\mu+1}$ now follows easily from condition (4) for $\mathcal{F}_{\mu}$.

\section{REFERENCES}

[1] J. Baker, Ph.D. Thesis, University of Wisconsin, 2001, to appear.

[2] A. Dow, Good and OK ultrafilters, Trans. Amer. Math. Soc. 290 (1985) 145-160. MR 86f:54044

[3] R. Engelking and M. Karłowicz, Some theorems of set theory and their topological consequences. Fund. Math. 57 (1965) 275-285. MR 33:4880

[4] H. J. Keisler, Good ideals in fields of sets, Ann. of Math. 79 (1964) 338-359. MR 29:3383

[5] K. Kunen, Ultrafilters and independent sets, Trans. Amer. Math. Soc. 172 (1972) 299-306. MR 47:3170

[6] K. Kunen, Weak P-points in $\mathbb{N}^{*}$, Colloq. Math. Soc. János Bolyai 23 (1980) 741-749. MR 82a:54046

[7] W. Rudin, Homogeneity problems in the theory of Cech compactifications. Duke Math. J. 23 (1956) 409-419. MR 18:324d

Department of Mathematics, University of Wisconsin, Madison, Wisconsin 57306

E-mail address: baker@math.wisc.edu

Department of Mathematics, University of Wisconsin, Madison, Wisconsin 57306

E-mail address: kunen@math.wisc.edu

$U R L:$ http://www. math.wisc.edu/ kunen 